\title{
FOTOCATÁLISIS HETEROGÉNEA PARA LA REMOCIÓN DE ANTIMONIO (Sb) EN AGUA UTILIZANDO DIÓXIDO DE TITANIO $\left(\mathrm{TiO}_{2}\right)$ COMO CATALIZADOR
}

Heterogeneous photocatalysis for the removal of antimony in water using titanium dioxide as a catalyst

Idalia ORRANTIA ${ }^{1}$, Damaris ACOSTA ${ }^{2 *}$, Luis SALINAS $^{1}$, Guillermo GONZÁLEZ $^{2}$ y Luis LOZOYA ${ }^{2}$

${ }^{1}$ Universidad Autónoma de Chihuahua, Facultad de Ciencias Químicas, Campus Universitario \# 2 Circuito Universitario, Chihuahua, Chih. C.P. 31125, México

${ }^{2}$ Centro de Investigación en Materiales Avanzados, Departamento de Medio Ambiente y Energía, Calle Miguel de Cervantes 120, Complejo Industrial, Chihuahua, Chih. C.P. 31136, México

*Autora para correspondencia damaris.acosta@cimav.edu.mx

(Recibido febrero 2018, aceptado mayo 2019)

Palabras clave: procesos de oxidación avanzada, procesos sustentables, fotocatalizadores, remoción de contaminantes

\section{RESUMEN}

El objetivo de esta investigación fue manipular el método de fotocatálisis heterogénea para remoción de antimonio $(\mathrm{Sb})$ en aguas industriales. Se utilizó el dióxido de titanio $\left(\mathrm{TiO}_{2}\right)$ como catalizador y peróxido de hidrógeno $\left(\mathrm{H}_{2} \mathrm{O}_{2}\right)$ como agente oxidante. El sistema fue conformado por un tanque de almacenamiento y un colector solar tubular plano el cual estuvo expuesto a la radiación en flujo continuo por varios tiempos de exposición, modificando la concentración del catalizador, agente oxidante y el pH de la solución. Los niveles de $\mathrm{Sb}$ esperados en la remoción fueron de $0.006 \mathrm{mg} / \mathrm{L}$ tomando como base los límites máximos permisibles de la Agencia de Protección Ambiental de los Estados Unidos de América (EPA) para agua potable. Se hicieron pruebas experimentales hasta encontrar los porcentajes más elevados de remoción. Los resultados obtenidos fueron de $74.2 \%$ a pH 7 con $1 \mathrm{~g}$ de $\mathrm{TiO}_{2}$ y $10 \mathrm{~mL}$ por h de $\mathrm{H}_{2} \mathrm{O}_{2}, 99.54 \%$ a pH 7 con $4 \mathrm{~g}$ de $\mathrm{TiO}_{2}, 86.72 \%$ a pH 4, con 3 g del $\mathrm{TiO}_{2}$, a 10,20 y 8 h de exposición respectivamente, con un rango de luz UV de 4 a 11 monitoreada de la estación meteorológica de la ciudad. Se concluye que a $\mathrm{pH}$ neutro con una cantidad de $4 \mathrm{~g}$ de $\mathrm{TiO}_{2}$, sin agregar el $\mathrm{H}_{2} \mathrm{O}_{2}$, en un tiempo de $20 \mathrm{~h}$, el sistema fotocatalítico desarrollado removió el $\mathrm{Sb}$ del agua tratada en concentraciones aceptables a la normatividad de agua potable de la EPA.

Key words: advanced oxidation processes, sustainable processes, solar collectors, photocatalysts, removal of pollutants

\begin{abstract}
The objective of this investigation was to manipulate the heterogeneous photocatalysis method for the removal of antimony ( $\mathrm{Sb}$ ) in industrial waters. Titanium dioxide $\left(\mathrm{TiO}_{2}\right)$ was used as a catalyst and hydrogen peroxide as the oxidant agent. The system consisted of a storage tank and a flat tubular solar collector which was exposed to the radiation in continuous flow for several exposure times, modifying the concentration
\end{abstract}


of the catalyst, oxidizing agent and the $\mathrm{pH}$ of the solution. The levels of Sb expected in the removal were $0.006 \mathrm{mg} / \mathrm{L}$ based on the maximum permissible limits of the United States Environmental Protection Agency (EPA) for drinking water. Experimental tests were made until finding the highest percentages of removal. The results obtained were $74.2 \%$ at $\mathrm{pH} 7$ with $1 \mathrm{~g}$ of $\mathrm{TiO}_{2}$ and $10 \mathrm{~mL}$ per h of $\mathrm{H}_{2} \mathrm{O}_{2}, 99.54 \%$ at $\mathrm{pH} 7$, with $4 \mathrm{~g}$ of $\mathrm{TiO}_{2}, 86.72 \%$ at $\mathrm{pH} 4$ with $3 \mathrm{~g}$ of $\mathrm{TiO}_{2}$, at 10,20 and $8 \mathrm{~h}$ of exposure respectively, with a UV light range of 4 to 11 monitored from the city's weather station. It is concluded that at neutral $\mathrm{pH}$ with an amount of $4 \mathrm{~g}$ of $\mathrm{TiO}_{2}$, without adding the $\mathrm{H}_{2} \mathrm{O}_{2}$, in a time of $20 \mathrm{~h}$, the developed photocatalytic system removed the $\mathrm{Sb}$ of the treated water in acceptable concentrations to the EPA's drinking water regulations.

\section{INTRODUCCIÓN}

El $71 \%$ de la superficie del planeta está conformada por agua, que es un recurso indispensable para la vida, pero limitado. El agua no sólo es necesaria para la naturaleza física de los seres vivos, sino que demás contribuye al desarrollo de diversas actividades humanas. Este recurso natural que puede proceder de fuentes superficiales como ríos, lagos y escorrentía, es objeto día a día de una severa contaminación, producto de las actividades del hombre. El efecto en ecosistemas acuáticos se traduce en la desaparición de la vegetación natural, disminuyendo la cantidad de oxígeno y produciendo la muerte de fauna acuática e incluso la afectación a la salud humana. La contaminación en el agua causada por la presencia de metales y metaloides representa, en la actualidad, uno de los problemas ambientales más importantes y preocupantes que ponen en riesgo la salud de la humanidad. Por ejemplo, uno de los elementos preocupantes en agua es el Sb. Este tiene una amplia variedad de aplicaciones como retardante de flama, en pinturas, cerámicos y plásticos (Belzile et al., 2011). Aunque no es un elemento abundante en la naturaleza, se puede encontrar principalmente combinado con diversos elementos, en especial en minerales que contienen estibina $\left(\mathrm{Sb}_{2} \mathrm{~S}_{3}\right)$, valentinita $\left(\mathrm{Sb}_{2} \mathrm{O}_{3}\right)$, kermesita $\left(\mathrm{Sb}_{2} \mathrm{~S}_{2} \mathrm{O}\right)$ y senarmontita $\left(\mathrm{Sb}_{2} \mathrm{O}_{3}\right)$. También es posible encontrar en la naturaleza los antimoniuros metálicos NiSb (breithaupita), NiSbS (ulmanita) y $\mathrm{Ag}_{2} \mathrm{Sb}$ (dicrasita). El Sb también puede provenir de fuentes antrópicas como la industria y la minería donde se extrae para su utilización en diversas aplicaciones.

El Sb se puede encontrar en varios estados de oxidación $(0, \mathrm{III}, \mathrm{V})$, pero el $\mathrm{Sb}(\mathrm{V})$ y el $\mathrm{Sb}$ (III) son predominantes en el ambiente, biológica y geoquímicamente. Los comportamientos toxicológicos y fisiológicos dependerán de su estado de oxidación. Por ejemplo, el Sb (III) muestra una alta afinidad para las células rojas de la sangre (OMS 2003). Generalmente los compuestos de $\mathrm{Sb}$ (III) son 10 veces más tóxicos que los del Sb (V) (Filella et al. 2007), lo que justifica la oxidación de éste antes de su tratamiento, además de permitirle combinarse fácilmente con otros compuestos.

La exposición del humano al $\mathrm{Sb}$ puede tener lugar por medio de la respiración, por consumo del agua potable y de los alimentos que lo contengan, pero también por contacto con suelos y otras sustancias. El tipo de Sb en el agua de consumo es determinante en su toxicidad; al parecer, el $\mathrm{Sb}$ procedente de materiales que lo contienen está en forma de oxoanión de $\mathrm{Sb}(\mathrm{V})$, que es la menos tóxica.

Las concentraciones de $\mathrm{Sb}$ que se disuelven en ríos y lagos son bajas, generalmente menores que cinco partes de $\mathrm{Sb}$ por 1 billón de partes de agua $(\mu \mathrm{g} / \mathrm{L})$. Por otro lado, las concentraciones de Sb encontradas en un río donde se vertieron desechos de la minería fueron tan altas como de $8 \mu \mathrm{g} / \mathrm{L}$ (ATSDR 1992). Las concentraciones más altas encontradas en sitios de desechos tóxicos y en los lugares donde se procesa el $\mathrm{Sb}$ oscilan entre 109 y $2,550 \mathrm{mg} / \mathrm{kg}$ (ATSR 1992)

$\mathrm{El} \mathrm{Sb}$ es regulado como un contaminante del agua potable, ya que puede causar efectos en la salud, como náuseas, vómitos y diarrea. La exposición a largo plazo puede conducir a un aumento de colesterol en la sangre y a la disminución de azúcar en la misma. La EPA no ha clasificado al $\mathrm{Sb}$ como un carcinógeno humano en agua debido a la falta de estudios. Sin embargo, las investigaciones muestran que el $\mathrm{Sb}$ y As, un carcinógeno comprobado, son igualmente tóxicos (Gebel, 1997). No hay experimentos con animales que permitan cuantificar el potencial cancerígeno de compuestos solubles o insolubles de Sb. El Centro Internacional de Investigadores sobre el cáncer (CIIC) ha concluido que el trióxido de $\mathrm{Sb}$ es posiblemente cancerígeno paraelserhumano. Hay algunos indicios de 
la capacidad cancerígena de ciertos compuestos de $\mathrm{Sb}$ por inhalación, pero no hay datos que indiquen su capacidad cancerígena por vía oral.

La ingesta oral diaria de $\mathrm{Sb}$ es, al parecer, significativamente mayor que la exposición por inhalación, aunque la exposición total procedente de fuentes ambientales, los alimentos y el agua de consumo es baja comparada con la exposición por motivos laborales (OMS 2003). Varios autores están de acuerdo en que la dosis letal media se sitúa entre 10 y $11.2 \mathrm{mg} / 100$ g (Nordberg, 2001). Otro punto importante sobre la ingesta de este metaloide es su liberación a partir del tereftalato de polietileno (PET) en productos alimenticios, dado que durante el almacenamiento hay un posible peligro para la salud de los seres humanos. En diferentes estudios se encontró que el Sb es liberado de los envases de PET de diferentes bebidas, agua y jugos (Shotyk 2006). Los límites máximos permisibles para el $\mathrm{Sb}$ en agua potable en diferentes países y organizaciones gubernamentales se encuentran desde $0.005 \mathrm{mg} / \mathrm{L}$ para Costa Rica, $0.006 \mathrm{mg} / \mathrm{L}$ para El Salvador, la EPA y la Sociedad Americana de Pruebas en Materiales (ASTM), $0.015 \mathrm{mg} / \mathrm{L}$ para Japón y de $0.02 \mathrm{mg} / \mathrm{L}$ para la Organización Mundial de la Salud (OMS), Brasil y Perú (OMS 2003).

Las técnicas más comunes para determinar el $\mathrm{Sb}$ en muestras ambientales incluyen la espectrometría de masas con plasma de acoplamiento inductivo (ICP-MS), la espectroscopia de absorción atómica con generador de hidruros y la espectroscopia de fluorescencia (Filella et al. 2009).

Con la finalidad de remover al $\mathrm{Sb}$ del agua se han implementado métodos tradicionales utilizados en la remoción del As por poseer un comportamiento químico similar, tales como la coagulación/floculación empleando sales de fierro, osmosis inversa, la biorremediación y adsorbentes como el carbón activado (Ungureanu et al. 2015). También se han probado métodos de fotooxidación del Sb (III) en presencia de diferentes especies de hierro (Fe) (III), ya que de manera natural en suelos y en agua la interacción del $\mathrm{Sb}$ con el Fe posiblemente es un proceso de control de intercambio del $\mathrm{Sb}$ (III) al Sb (V), siendo este último menos peligroso (Kong 2016).

Entre otros métodos conocidos para la remoción de metales y metaloides como el Sb en agua, están los procesos de oxidación avanzada, donde se incluye la fotocatálisis heterogénea empleando $\mathrm{TiO}_{2}$ como catalizador. El proceso fotocatalítico del $\mathrm{TiO}_{2}$ tiene lugar a partir de la irradiación del mismo con luz, de una energía suficiente como para igualar o superar la brecha energética del semiconductor, produciendo la excitación de un electrón de la banda de valencia
(BV) a la banda de conducción (BC). De este modo (ver fórmulas abajo) se crean pares electrón-hueco $(\mathrm{e} / \mathrm{h}+)(\mathrm{a})$, que pueden migrar a la superficie del catalizador, quedando atrapadas en sitios superficiales (b y c) y reaccionar con las especies adsorbidas ( $\mathrm{d}$ y e). Los huecos que se forman en la banda de valencia del sólido, reaccionan con especies dadoras de electrones, como moléculas de agua o iones hidroxilos unidos a la superficie del catalizador, generando los radicales hidroxilos $(\mathrm{OH})$, principales responsables de la degradación de la materia orgánica. A su vez, los electrones que llegan a la banda de conducción, pueden reaccionar con una especie aceptora como el $\mathrm{O}_{2}$, dando lugar a radicales $\mathrm{O}_{2}{ }^{-}$, que participan adicionalmente en la oxidación de la materia orgánica. No obstante, en competencia con los procesos de transferencia de carga, tienen lugar los procesos de recombinación (f y g), en los que el par electrón-hueco se recombina antes de reaccionar con las especies adsorbidas en la superficie catalítica (Carbajo 2013).

a) $\mathrm{TiO}_{2}+$ h.v. $\rightarrow \mathrm{e}^{-}{ }_{\mathrm{BC}}+\mathrm{h}^{+}{ }_{\mathrm{BV}}$

b) $\mathrm{h}^{+}{ }_{\mathrm{BV}}+>\mathrm{Ti}^{\mathrm{IV}} \mathrm{OH} \rightarrow\left\{>\mathrm{Ti}^{\mathrm{IV}} \mathrm{OH}^{\bullet}\right\}+$

c) $\mathrm{e}^{-}{ }_{\mathrm{BC}}+>\mathrm{Ti}^{\mathrm{IV}} \mathrm{OH} \rightarrow\left\{\mathrm{Ti}^{\mathrm{III}} \mathrm{OH}^{*}\right\}$

d) $\left\{>\mathrm{Ti}^{i V} \mathrm{OH}^{\bullet}\right\}++\operatorname{Red} \rightarrow>\mathrm{Ti}^{\mathrm{IV}} \mathrm{OH}+\mathrm{Red}^{\bullet+}$

e) $\mathrm{e}^{-}{ }_{\mathrm{BC}}+\mathrm{Ox}+\rightarrow>\mathrm{Ti}^{\mathrm{iV}} \mathrm{OH}+\mathrm{Ox}^{\cdot-}$

f) $\mathrm{e}^{-}{ }_{\mathrm{BC}}+\left\{>\mathrm{Ti}^{\mathrm{IV}} \mathrm{OH}^{\bullet}\right\}+\rightarrow>\mathrm{Ti}^{\mathrm{IV}} \mathrm{OH}$

g) $\mathrm{h}_{\mathrm{BV}}^{+}+\left\{\mathrm{Ti}^{\mathrm{III}} \mathrm{OH}\right\} \rightarrow>\mathrm{Ti}^{\mathrm{IV}} \mathrm{OH}$

En esta investigación se hipotetizó la opción viable de la oxidación del Sb (III) a Sb (V) a través de los $\mathrm{OH}^{*}$ y los huecos de electrón:

$\mathrm{Sb}^{3}+\mathrm{H}_{2} \mathrm{O} \frac{\mathrm{TiO}_{2}}{h v}>\left(\mathrm{SbO}_{4}\right)^{-3}+5 \mathrm{e}^{-}$

El objetivo de este estudio fue utilizar el proceso llamado fotocatálisis heterogénea, con dióxido de titanio $\left(\mathrm{TiO}_{2}\right)$ como catalizador y peróxido de hidrogeno $\left(\mathrm{H}_{2} \mathrm{O}_{2}\right)$ como agente oxidante a flujo continuo expuesto a luz solar en diferentes tiempos, para remover $\mathrm{Sb}$ de una muestra sintética siguiendo el principio de fotocatálisis donde el $\mathrm{TiO}_{2}$ actúa oxidando el $\mathrm{Sb}$ (III) a Sb (V), siendo este último precipitado fácilmente con sales de cloruro férrico $\left(\mathrm{FeCl}_{3}\right)$.

\section{MATERIALES Y MÉTODOS}

El desarrollo experimental fue guiado por los siguientes ocho pasos y las subsecuentes etapas:

1) diseño del reactor solar piloto; 2) establecimiento de concentraciones (contaminante, catalizador, agente oxidante); 3 ) establecimiento de tiempo 
de exposición; 4) identificación de parámetros de medición; 5) cuantificación de la degradación por medio de espectrofotometría de absorción atómica en flama; 6) cuantificación de la degradación por medio de generación de hidruros; 7) adición de $\mathrm{FeCl}_{3}$ para la remoción y 8) cuantificación de la remoción por generador de hidruros.

\section{Construcción del sistema fotocatalítico}

El sistema fotocatalítico fue diseñado con un tanque de alimentación recirculación de 19 L, donde se preparó la muestra; en este tanque se descargaba la tubería proveniente de la reacción, y para generar la recirculación de la solución tratada se conectó el otro extremo a una bomba de agua con capacidad de $6 \mathrm{~L} / \mathrm{min}$, permitiendo así un flujo continuo. El volumen al que se le dio tratamiento fue de $1.6 \mathrm{~L}$ en un lapso de $50 \mathrm{~s}$.

\section{Parámetros de operación}

Para encontrar las condiciones más favorables de remoción del $\mathrm{Sb}$ a través del proceso de fotocatálisis heterogénea se modificaron las variables de concentración del catalizador $\left(\mathrm{TiO}_{2}\right)$ empleando $0.5,1,2,3$, 3.5 y 4 g, los volúmenes de $\mathrm{H}_{2} \mathrm{O}_{2}$ fueron 5,10 y 20 $\mathrm{mL}$ por hora, el $\mathrm{pH}$ obtenido fue de 3,4 y 7 unidades, ajustando la solución a $\mathrm{pH}$ ácido con ácido sulfúrico 0.1 molar $\left(\mathrm{H}_{2} \mathrm{SO}_{4} 0.1 \mathrm{M}\right)$. Se estableció un tiempo de exposición a la luz solar de $7 \mathrm{~h}$ diarias, pudiendo alcanzar hasta $21 \mathrm{~h}$ en un plazo de tres días, realizando la toma de muestra al inicio y a lo largo del tiempo del tratamiento. Para la remoción del $\mathrm{Sb}$ restante en la solución tratada se utilizó 0.1 y $0.2 \mathrm{~g} / \mathrm{L}$ de $\mathrm{FeCl}_{3}$ para su precipitación.

Las modificaciones realizadas para cada experimento se definieron con base en los resultados de remoción obtenidos y por ensayos de prueba y error, como técnica exploratoria para encontrar los resultados esperados.

\section{Elaboración de la muestra fortificada}

De acuerdo con la capacidad del sistema fotocatalítico, se planteó un volumen de muestra de $6 \mathrm{~L}$, empleando una solución fortificada de $\mathrm{Sb}$ de $1 \mathrm{mg} / \mathrm{L}$ como concentración de referencia. La solución madre de $\mathrm{Sb}$ empleada para preparar la muestra fue marca High Purity de $1000 \mathrm{mg} / \mathrm{L}$ y el agua utilizada para preparar las soluciones sintéticas fue agua de la red del laboratorio de residuos del CIMAV-Chihuahua.

\section{Parámetros de medición}

Se modificaron los parámetros $\mathrm{pH}$, concentración del catalizador y del agente oxidante y se registró la radiación solar, temperatura y turbiedad de cada experimento, con la finalidad de encontrar cuales ensayos presentaron las mejores condiciones de remoción del $\mathrm{Sb}$.

\section{Desarrollo experimental}

Se montó el sistema fotocatalítico colocando la superficie reflectante y la tubería flexible (área de reacción). Enseguida se preparó la muestra en el tanque de almacenamiento, añadiendo el catalizador, agitando manualmente para incorporarlo en la solución de Sb. Para finalizar se puso en funcionamiento expuesto a la radiación solar. Las pruebas se realizaron entre las 9:00 y las 16:00 h con el fin de aprovechar la mayor cantidad de radiación solar en la ciudad. Se tomó una muestra al inicio del proceso y las demás a lo largo del mismo midiendo $\mathrm{pH}$ y temperatura, además se registró la radiación UV reportada de la página https://www.wunderground.com/ de la ciudad de Chihuahua, Chihuahua. La medición del Sb se realizó por la técnica de espectrofotometría de absorción atómica en flama; si la concentración no era detectable se procedió a hacer el análisis en generador de hidruros. Para remover el $\mathrm{TiO}_{2}$ se dejó sedimentar y enseguida se filtró adicionando como etapa final $\mathrm{FeCl}_{3}$ para precipitar el $\mathrm{Sb}$ restante en la solución. Las pruebas se realizaron por duplicado y fueron agrupadas en tres categorías: pruebas con agente oxidante y catalizador (casos 1, 2, 3 y 4) ensayos con catalizador y $\mathrm{pH}$ neutro (casos 5 y 6 ) y con catalizador y pH ácido (casos 7 y 8 ).

\section{RESULTADOS}

En el Cuadro I, se presenta un resumen de las condiciones y resultados de remoción del $\mathrm{Sb}$ en el proceso de fotocatálisis heterogénea aplicado en la presente investigación.

La intensidad de radiación ultravioleta recibida mientras se corrieron las pruebas de fotocatálisis se encontró en un rango de 3 a 11, considerando favorables días sin nubosidad y de temperaturas mayores a $\operatorname{los} 20^{\circ} \mathrm{C}$.

El tratamiento con $\mathrm{FeCl}_{3}$ se aplicó como complemento a la experimentación una vez tratada la solución con el $\mathrm{TiO}_{2}$ ya que se realizó como prueba inicial antes del proceso de fotocatálisis heterogénea y no se observó ninguna precipitación ni disminución de los niveles de $\mathrm{Sb}$, de estos casos no se presentan resultados.

En la figura 1 se presentan las cinéticas de reacción para cada uno de los casos descritos (ver Cuadro I). 
CUADRO I. RESUMEN DE LAS CONDICIONES Y RESULTADOS DE LA EXPERIMENTACIÓN EN LA REMOCIÓN DEL Sb

\begin{tabular}{|c|c|c|c|c|c|c|c|c|}
\hline Caso & 1 & 2 & 3 & 4 & 5 & 6 & 7 & 8 \\
\hline Tipo del Sb removido & $\mathrm{Sb}$ (III) & $\mathrm{Sb}$ (III) & $\mathrm{Sb}$ (III) & $\mathrm{Sb}$ (III) & $\mathrm{Sb}$ (III) & $\mathrm{Sb}$ (III) & $\mathrm{Sb}$ (III) & $\mathrm{Sb}$ (III) \\
\hline Concentración inicial del Sb & $1 \mathrm{mg} / \mathrm{L}$ & $1 \mathrm{mg} / \mathrm{L}$ & $1 \mathrm{mg} / \mathrm{L}$ & $1 \mathrm{mg} / \mathrm{L}$ & $1 \mathrm{mg} / \mathrm{L}$ & $1 \mathrm{mg} / \mathrm{L}$ & $1 \mathrm{mg} / \mathrm{L}$ & $1 \mathrm{mg} / \mathrm{L}$ \\
\hline Concentración final del Sb & $0.857 \mathrm{mg} / \mathrm{L}$ & $0.258 \mathrm{mg} / \mathrm{L}$ & $0.767 \mathrm{mg} / \mathrm{L}$ & $0.82 \mathrm{mg} / \mathrm{L}$ & $0.0045 \mathrm{mg}(\mathrm{L}$ & $0.899 \mathrm{mg} / \mathrm{L}$ & $0.0153 \mathrm{mg} / \mathrm{L}$ & $0.0132 \mathrm{mg} / \mathrm{L}$ \\
\hline Cantidad de $\mathrm{TiO}_{2}$ & $1 \mathrm{~g}$ & $1 \mathrm{~g}$ & $1 \mathrm{~g}$ & $0.5 \mathrm{~g}$ & $4 \mathrm{~g}$ & $3.5 \mathrm{~g}$ & $2 \mathrm{~g}$ & $3 \mathrm{~g}$ \\
\hline Cantidad de $\mathrm{H}_{2} \mathrm{O}_{2}$ & $5 \mathrm{~mL} / \mathrm{h}$ & $10 \mathrm{~mL} / \mathrm{h}$ & $20 \mathrm{~mL} / \mathrm{h}$ & $20 \mathrm{~mL} / \mathrm{h}$ & -------- & ------------ & ---------- & --------- \\
\hline Cantidad de $\mathrm{FeCl}_{3}$ & $0.2 \mathrm{~g} / \mathrm{L}$ & $0.2 \mathrm{~g} / \mathrm{L}$ & $0.2 \mathrm{~g} / \mathrm{L}$ & $0.2 \mathrm{~g} / \mathrm{L}$ & $0.2 \mathrm{~g} / \mathrm{L}$ & $0.2 \mathrm{~g} / \mathrm{L}$ & $0.2 \mathrm{~g} / \mathrm{L}$ & $0.2 \mathrm{~g} / \mathrm{L}$ \\
\hline $\mathrm{pH}$ inicial & 7.50 & 6.50 & 6.64 & 6.70 & 7.03 & 6.60 & 3.30 & 3.90 \\
\hline Tiempo de exposición & $10 \mathrm{~h}$ & $10 \mathrm{~h}$ & $10 \mathrm{~h}$ & $12 \mathrm{~h}$ & $20 \mathrm{~h}$ & $11 \mathrm{~h}$ & $3 \mathrm{~h}$ & $8 \mathrm{~h}$ \\
\hline Eficiencia de remoción & $14.3 \%$ & $74.2 \%$ & $23.3 \%$ & $18 \%$ & $99.5 \%$ & $91 \%$ & $84.6 \%$ & $86.7 \%$ \\
\hline Cumple con normatividad & - & - & ------ & 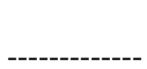 & $\begin{array}{l}\text { EPA y } \\
\text { ASTM }\end{array}$ & - & & \\
\hline & & & & & $0.006 \mathrm{mg} / \mathrm{L})$ & & & \\
\hline
\end{tabular}

Nota: La línea punteada $=$ No aplica

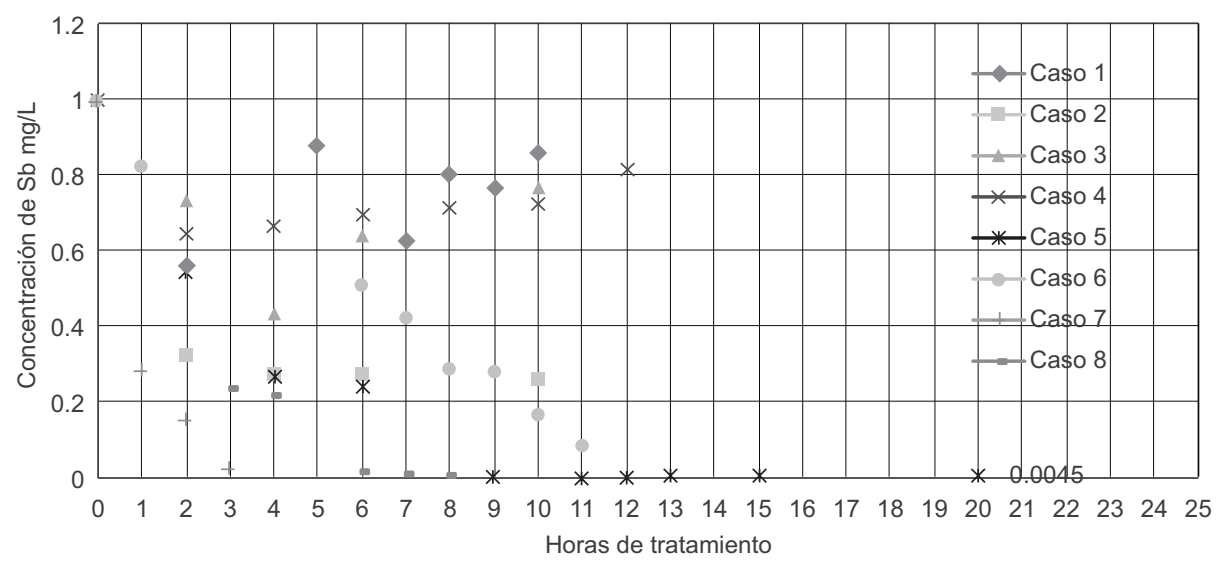

Fig. 1. Cinéticas de reacción del $\mathrm{Sb}$ en el tratamiento de fotocatálisis heterogénea

Todos los experimentos iniciaron en $1 \mathrm{mg} / \mathrm{L}$ de $\mathrm{Sb}$ y a través del tiempo del tratamiento la concentración se fue modificando. En el caso 5 fue donde se obtuvo el mejor porcentaje de remoción.

\section{DISCUSIÓN}

Con esta investigación se demuestra que el tiempo de exposición, la concentración del catalizador, el volumen del oxidante y el $\mathrm{pH}$ son factores que influyen en gran medida al proceso de fotocatálisis heterogénea para remover contaminantes del agua ya sea como tratamiento único o como parte de varias metodologías para remoción de $\mathrm{Sb}$ en este medio. Cordova (2013) realizó un procedimiento para remover As (metaloide similar al $\mathrm{Sb}$ ) de agua empleando $\mathrm{TiO}_{2}$, diferentes concentraciones de $\mathrm{Fe}$ (III), modificando el pH y tiempo de exposición, sin embargo, su muestra fue tratada en botellas de PET (polietileno) impregnadas con el catalizador $\left(\mathrm{TiO}_{2}\right)$, no en un colector solar como se trató el $\mathrm{Sb}$ en este estudio.

Diferentes autores han investigado el comportamiento del $\mathrm{Sb}$ en contacto con el $\mathrm{FeCl}_{3}$ y el hidróxido de hierro encontrando que estas sales son capaces de foto oxidar el Sb (III) a Sb (V) (Kong 2016). Sin embargo, en el presente estudio el tratamiento con $\mathrm{FeCl}_{3}$ se utilizó como complemento al tratamiento de fotocatálisis heterogénea con la finalidad de precipitar el Sb residual en la solución. Xu et al. (2011) compararon la capacidad y el mecanismo de remoción de $\mathrm{Sb}$, del óxido binario de fierro y manganeso, del hidróxido férrico y del dióxido de manganeso, encontrando que el primero quizá sea un compuesto prometedor para remover $\mathrm{Sb}$ de agua potable y agua residual.

Es conocido que el $\mathrm{pH}$ afecta también a la movilidad de los metales y metaloides (Caviedes et al. 2015) en el agua al igual que las propiedades superficiales 
de un catalizador y a la forma química del compuesto a degradar, y ello se manifiesta en alteraciones de la velocidad de degradación y en la tendencia a la floculación del catalizador. Por lo tanto, se logró observar que a menor $\mathrm{pH}$ mayor rapidez de degradación del $\mathrm{Sb}$. Además, es importante mencionar que el $\mathrm{TiO}_{2}$ es un anfótero y, por ello, adsorbe algunas especies químicas dependiendo del $\mathrm{pH}$ en el cual se encuentre. También cabe destacar que la cantidad del catalizador mejora la degradación del contaminante, teniendo en cuenta la óptica del reactor, la naturaleza de la especie a tratar y la disponibilidad de fotones en el sistema, por lo que el colector solar plano tubular utilizado aprovechó adecuadamente la radiación solar directa y difusa recibida en el periodo de la realización del presente trabajo.

En cada caso de la experimentación se hicieron varias modificaciones tratando de encontrar las mejores condiciones de remoción. En los casos 1, 3 y 4 se puso en contacto al catalizador y al oxidante en la solución de $\mathrm{Sb}$, originando una posible competencia por la superficie de adsorción, dando como resultado bajos porcentajes de degradación. En el caso 2 se obtuvo un mejor porcentaje de remoción al aumentar el oxidante en el mismo tiempo de contacto que los casos 1 y 3 , sin embargo, el $\mathrm{pH}$ fue menor generando mayor movilidad del metaloide seguido de una mayor disminución del contaminante. Debido a que se apreció un comportamiento desfavorable entre el catalizador y el oxidante a $\mathrm{pH}$ menor a 7, para los casos 5, 6, 7 y 8 se descartó el uso del $\mathrm{H}_{2} \mathrm{O}_{2}$ y se continuó modificando la cantidad de $\mathrm{TiO}_{2}$ y los tiempos de tratamiento, obteniendo el mejor porcentaje de remoción en el caso 5. Sin embargo, el tiempo empleado fue mayor que para los casos 6, 7 y 8 además de que la cantidad del catalizador originó turbidez en la solución muy próxima al límite de turbidez en agua de 5 unidades nefelométricas de turbidez (NTU). Por este motivo los experimentos consecutivos se trataron con menor cantidad de $\mathrm{TiO}_{2}$ y los casos 7 y 8 en condiciones ácidas, lo cual resultó favorable en tiempo y porcentaje de remoción. Sin embargo, uno de los objetivos de este trabajo fue ofrecer un tratamiento amigable con el ambiente para eliminar contaminantes. Por esta razón, se consideró como el mejor experimento aquel que tuviera una remoción por encima del $90 \%$ en condiciones de $\mathrm{pH}$ cercanas a la neutralidad.

De los resultados obtenidos se concluye que el tratamiento de fotocatálisis heterogénea para remoción de $\mathrm{Sb}$ en agua fue efectivo, ya que al estar en contacto con el $\mathrm{TiO}_{2}$ en exposición a la radiación solar se oxida el $\mathrm{Sb}$ (III) al $\mathrm{Sb}$ (V) a través de los $\mathrm{OH}^{\circ}$ y los huecos de electrón de acuerdo con la reacción hipotética planteada, facilitando su extracción de la solución y posterior precipitación con el $\mathrm{FeCl}_{3}$.

\section{AGRADECIMIENTOS}

Al técnico Luis de la Torre y a la Dra. Virginia Collins del Laboratorio de catálisis, fotocatálisis y electrocatálisis del Centro de Investigación en Materiales Avanzados por su apoyo y colaboración en todo momento del desarrollo de esta investigación.

\section{REFERENCIAS}

ATSDR (1992). Resumen de Salud Pública Antimonio CAS\#: 7440-36-0. Agencia para Sustancias Tóxicas y el Registro de Enfermedades. Reseña toxicológica del antimonio, Atlanta, GA: Departamento de salud y servicios humanos de los E.E.U.U. servicio de Salud Pública, 1-6pp.

Belzile N., Chen Y. y Filella M., (2011). Human Exposure to Antimony: Sources and Intake. Critical reviews in environmental science and technology 41:14, 13091373. DOI: $10.1080 / 10643381003608227$.

Carbajo J., (2013). Aplicación de la fotocatálisis solar a la degradación de contaminantes orgánicos en fase acuosa con catalizadores nanoestructurados de $\mathrm{TiO}_{2}$. Tesis Doctoral. Facultad de Ciencias. Universidad Autónoma de Madrid. Madrid, España, 277 pp.

Caviedes D.I., Muñoz, R. A. Perdomo, A., Rodríguez D. y Sandoval I.J., (2015). Tratamientos para la Remoción de Metales Pesados Comúnmente Presentes en Aguas Residuales Industriales. Una Revisión. Revista Ingeniería y Región 13. 73-90.

Cordova L., (2013). Desinfección y remoción de arsénico del agua por fotocatálisis heterogénea. Tesis de maestría. Centro de Investigación en Materiales Avanzados S.C. Chihuahua. Chih. México. 156 pp.

Filella M., Belzile y N., Lett, M. C., (2007). Antimony in the environment: A review focused on natural waters. III. Microbiota relevant interactions. Earth-Science Reviews 80, 195-217.

Filella M., Williams P.A. y Belzile N., (2009) Antimony in the environment: knows and unknowns. Journal of Environmental Chemistry 6, 95-105. DOI: 10.1071/EN09007.

Gebel T., (1997). Arsenic and antimony: comparative approach on mechanistic toxicology. Chem. Biol. Interact. 107(3), 131-144.

Kong L., He M. y Hu X., (2016). Rapid photooxidation of $\mathrm{Sb}$ (III) in the presence of different $\mathrm{Fe}$ (III) species. Geochimica et Cosmochimica Acta 180. 214-226. 
Nordberg G., (2001). Metales: Propiedades químicas y toxicidad. En: Enciclopedia de Salud y Seguridad en el Trabajo (Ministerio de Trabajo y Asuntos Sociales Subdirección General de Publicaciones). Gestión editorial Chantal Dufresne, BA. Madrid. España. pp. 63.01-63.75

OMS, (2003). Antimony in drinking-water. Organización Mundial de la Salud. Documento de referencia para la elaboración de las Guías de la OMS para la calidad del agua potable Ginebra, Suiza, 22pp.

Shotyk W., Krachler, M., y Chen, B., (2006). Contamination of Canadian and European bottled waters with antimony from PET containers. Journal of Environmental Monitoring 8, 288-292. DOI:10.1039/b517844b.
Ungureanu G., Santos S., Boaventura R. y Botelho C., (2015). Arsenic and antimony in water and wastewater: Overview of removal techniques with special reference to latest advances in adsorption. Journal of Environmental Management 151, 326-342. DOI: 10.1016/j.jenvman.2014.12.051.

Xu W., Wang H., Liu R., Zhao X. y Qu J., (2011). The mechanism of antimony(III) removal and its reactions on the surfaces of Fe-Mn Binary Oxide. Journal of colloid and interface science 363, 320-326. 